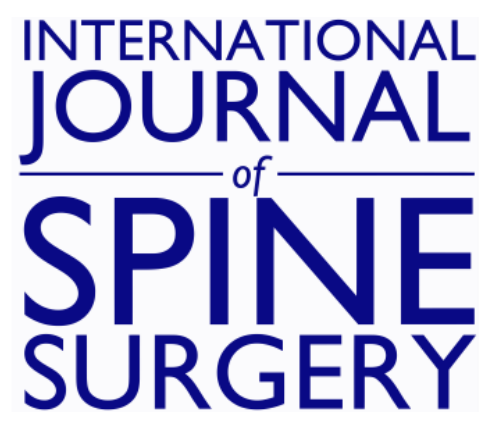

\title{
The Impact of Osteobiologic Subtype Selection on Perioperative Complications and Hospital-Reported Charges in Single- and Multi-Level Lumbar Spinal Fusion
}

Shane Shahrestani, Alexander M. Ballatori, Xiao Chen, Andy Ton, Jeffrey C. Wang and Zorica Buser

Int J Spine Surg 2021, 15 (4) 654-662

doi: https://doi.org/10.14444/8086

http://ijssurgery.com/content/15/4/654

This information is current as of April 26, 2023.

Email Alerts Receive free email-alerts when new articles cite this article. Sign up at:

http://ijssurgery.com/alerts

The International Journal of Spine Surgery

2397 Waterbury Circle, Suite 1,

Aurora, IL 60504, Phone: +1-630-375-1432 


\title{
The Impact of Osteobiologic Subtype Selection on Perioperative Complications and Hospital-Reported Charges in Single- and Multi-Level Lumbar Spinal Fusion
}

\author{
SHANE SHAHRESTANI, MS,${ }^{1,2}$ ALEXANDER M. BALLATORI, BA, ${ }^{1}$ XIAO CHEN, BA, ${ }^{1}$ ANDY TON, BS, ${ }^{1}$ \\ JEFFREY C. WANG, MD ${ }^{1}$ ZORICA BUSER, PHD ${ }^{1}$ \\ ${ }^{I}$ Department of Orthopaedic Surgery, Keck School of Medicine, University of Southern California, Los Angeles, California, ${ }^{2}$ Department of Medical Engineering, \\ California Institute of Technology, Pasadena, California
}

\begin{abstract}
Background: Over the last several decades, various osteobiologics including allograft, synthetics, and growth factors have been used for lumbar spinal fusion surgery. However, the data on these osteobiologic products remain controversial with conflicting evidence in the literature. This study evaluates the influence of osteobiologic type selection on perioperative complications and hospital-reported charges in single-level and multilevel lumbar fusion.

Methods: Using the 2016 and 2017 Nationwide Readmission Database, we conducted a retrospective cohort analysis of 125,143 patients who received lumbar fusion with either autologous tissue substitute, nonautologous tissue substitute, or synthetic substitute. This cohort was split into single-level and multilevel fusion procedures, and one-toone age and sex propensity score matching was implemented. This resulted in cohorts each consisting of 1967 patients for single-level fusion, and cohorts each consisting of 1657 patients for multilevel fusion. Statistical analysis included one-way analysis of variance and Tukey multiple comparisons of means.

Results: Autologous single-level fusion resulted in significantly more postoperative pain at 30-, 90-, and 180-day follow-up compared to fusion with nonautologous graft $(P<.05)$. Multilevel fusion with autologous graft had higher rates of acute postsurgical anemia compared with synthetic $(P=.021)$ and nonautologous $(P=.016)$ alternatives, and less postsurgical infection when compared with nonautologous fusion $(P=.0020)$. In addition, procedures using autologous osteobiologics were associated with significantly more neurological complications at 30 days $(P=.049)$ and 90 days $(P=.048)$ for multi-level fusion and at 30 days $(P=.044)$ for single-level fusion compared with the nonautologous group. Lastly, for both cohorts, the total accrued inpatient hospital charges during admission for patients receiving nonautologous grafts were the most expensive and those for patients receiving autologous grafts were the least expensive.

Conclusion: Significant differences were found between the groups with respect to rates of complications, including infection, postoperative pain, and neurologic injury. Furthermore, the hospital-reported charges of each procedure varied significantly. As the field of biologics continues to expand, it is important to continually evaluate the safety, efficacy, and cost-effectiveness of these novel materials and techniques.
\end{abstract}

Level of Evidence: 3

Clinical Relevance: With increased utilization of osteobiologics and spinal fusion being a common procedure, longitudinal data on readmissions, and post-operative complications are critical in guiding evidence-based practice.

Biologics

Keywords: lumbar fusion, osteobiologic, autologous, nonautologous, synthetic, multi-level, single-level

\section{INTRODUCTION}

Lumbar spine fusion is often the surgical solution of choice when treating various degenerative spinal conditions. The last several decades have seen a sharp increase in the use of spinal osteobiologics for lumbar fusion procedures, and many novel osteobiologic materials and techniques have been developed to promote vertebral fusion., ${ }^{1,2}$ These osteobiologics include, but are not limited to, autologous tissue substitutes, nonautologous tissue substitutes such as demineralized bone matrix, growth factors, small peptides, and synthetic substitutes. However, data outlining the properties of these osteobiologic products remain controversial due to conflicting evidence in the literature. Studies outlining the safety, efficacy, and costs associated with different osteobiologic subtypes vary widely, with little longitudinal outcome data. ${ }^{3-5}$ 
Manipulating the complex and multifaceted biological process of spine fusion demands multiple considerations with respect to the properties of each graft, including osteoconductive and/or osteoinductive abilities, biodegradability, porosity, mechanical properties, safety, and biocompatibility. ${ }^{6}$ Autologous osteobiologics involve harvesting the patient's own tissue, which is associated with complication rates of up to $19 \%$ including pain, infection, fracture, hematoma, neurological injury, and cosmetic deformity. ${ }^{7}$ Autograft remains the gold standard, however, due to its intrinsic biological properties, immunocompatibility, and fusion rates consistently over $90 \%$ in posterolateral lumbar spine fusion. ${ }^{8,9}$

Allografts and synthetic grafts present an opportunity to avoid donor site morbidity. Disadvantages of allograft usage include possible immunogenicity, low structural integrity, increased chances of poor incorporation into bone, and possible viral disease transmission of HIV, hepatitis B, and hepatitis $\mathrm{C},{ }^{10,11}$ though this complication is rare due to mandatory donor screening protocols. Allografts are commonly obtained from cadaveric donors and are osteoconductive but may lose a significant portion of their osteoinductive properties during processing. There has been a recent upwards trend toward using cell-based allografts, but the literature on this topic is heterogenous, with different cell preparation methods, surgical approaches, and comparator groups. ${ }^{12}$ Another biologic that has become increasingly used is the use of bone marrow aspirate, which has been shown in a recent systematic review to have similar fusion rates compared with autograft, though the total body of evidence is limited. ${ }^{13}$ Overall, allografts are more widely available and particularly advantageous to use in patients with poor bone but are often associated with far lower and less consistent fusion rates than autografts. ${ }^{14}$

Synthetic osteobiologics include ceramic glass, hydroxyapatite, calcium sulfate, and calcium phosphate. However, data on synthetics are inconclusive due to the small sample size in studies, no comparators and poor biomechanical support. ${ }^{1}$ Other graft materials not classified in the previous 3 subgroups such as recombinant human bone morphogenetic protein (rhBMP) have been shown to achieve fusion rates comparable with those of autografts, ${ }^{15}$ particularly when combined with an allograft, ${ }^{16}$ but may be a greater cost burden to hospitals. ${ }^{17}$ The rhBMP family has osteoinductive properties, though usage remains controversial and there are safety concerns regarding the side effect profile and potential for increased complications. ${ }^{18-20}$

Several systematic reviews have suggested that the various osteobiologic subtypes boast comparable effectiveness, with significantly different costs varying by procedure. ${ }^{5,21}$ Yet, large cohort studies that evaluate the accuracy of this finding are absent in the literature. As the use of osteobiologics for lumbar fusion continues to grow, clinical evidence demonstrating procedural efficacy and patient safety are required. With lumbar fusion becoming an increasingly more common procedure, longitudinal data that outline readmission frequency, postsurgical complications, and procedural efficacy are critical in guiding evidence-based practice. Here, we evaluate the effect of single- and multilevel lumbar fusion osteobiologic subtype on hospitalreported charges and complication rates at 30-, 90-, and 180-day readmission intervals.

\section{METHODS}

\section{Data Source}

For our analysis, we used the Nationwide Readmission Database (NRD) from 2016 and 2017. The NRD is a large yearly database provided by the Healthcare Cost and Utilization Project and the Agency for Healthcare Research and Quality that encompasses roughly $60 \%$ of the United States, with hospital readmission information for all ages and more than 17000000 discharges per year. These databases have been created to provide nationally representative information regarding inpatient hospital stays, readmissions, and their associated hospital-reported charges in the United States. Diagnoses and procedures are coded within each patient admission or readmission as International Classification of Diseases, Tenth Revision (ICD-10) codes. $^{22}$ NRD years 2015 and earlier were excluded due to their usage of ICD-9 coding (ICD10 usage became mandatory in late 2015), which does not have specific codes for each type of osteobiologic.

\section{Patient Selection}

All inpatient hospitalizations involving a lumbar fusion procedure with osteobiologic use were compiled to form a data set using the ICD-10 codes outlined in Table 1. Demographics and hospital information were collected for each inpatient stay. 
Table 1. Patient selection and complication International Classification of Diseases, Tenth Revision codes.

\begin{tabular}{ll}
\hline Procedure & \multicolumn{1}{c}{ Complication Code } \\
\hline Autologous one-level & 0SG0070, 0SG0071, 0SG007J, 0SG0370, 0SG0371, 0SG037J, 0SG0470, 0SG0471, 0SG047J \\
Autologous multilevel & 0SG1070, 0SG1071, 0SG107J, 0SG1370, 0SG1371, 0SG137J, 0SG1470, 0SG1471, 0SG147J \\
Synthetic one-level & 0SG00J0, 0SG00J1, 0SG00JJ, 0SG03J0, 0SG03J1, 0SG03JJ, 0SG04J0, 0SG04J1, 0SG04JJ \\
Synthetic multilevel & 0SG10J0, 0SG10J1, 0SG10JJ, 0SG13J0, 0SG13J1, 0SG13JJ, 0SG14J0, 0SG14J1, 0SG14JJ \\
Nonautologous one-level & 0SG00K0, 0SG00K1, 0SG00KJ, 0SG03K0, 0SG03K1, 0SG03KJ, 0SG04K0, 0SG04K1, 0SG04KJ \\
Nonautologous multilevel & 0SG10K0, 0SG10K1, 0SG10KJ, 0SG13K0, 0SG13K1, 0SG13KJ, 0SG14K0, 0SG14K1, 0SG14KJ \\
Infection & T814XXA, K6811, T814XXA, T8579XA, T80219A, T80211A, T80212A, T8022XA, T8029XA, T880XXA, \\
& A419, R6520, K6811, K6811, T8130XA, T8132XA, T8131XA, T8189XA,T8183XA, T8579XA, T8460XA, \\
& T847XXA, K6811, T8579XA, A419, R6520, T8130XA,T8132XA, T8131XA, T8133XA, T8183XA, \\
Pneumonia & T847XXA \\
Postoperative pain & J189 \\
Hardware malfunction & G8918, G8928 \\
& T84216A, T84226A, T84296A, T84318A, T84398A, T84428A, T84498A, T8459XA, T8459XD, T8460XA, \\
UTI & T8463XD, T8469XA, T847XXA, T847XXD, T8481XA, T8484XA, T8485XA, T8489XA \\
Neurological injury & N390 \\
& G9781: Intraoperative complications of nervous system \\
& G9782: Postprocedural complications and disorders of nervous system \\
& G97811, I97821: Intra- and postoperative cerebral infarction \\
\hline
\end{tabular}

Abbreviations: CSF, cerebrospinal fluid; UTI, urinary tract infection.

Patients were then separated into 2 groups based on whether they received a single-level spinal fusion or multilevel spinal fusion, then split again based on osteobiologic subtype, which included autologous, nonautologous, and synthetic graft material. Oneto-one propensity score matching for age and sex was then implemented to ensure that each group contained similar demographics and patient numbers for analysis. Readmissions were analyzed in 30-, 90-, and 180-day intervals. Elective readmissions were excluded from readmission analysis, as only nonelective readmissions and their associated complications were of interest. Autologous graft material included both iliac and local bone grafts, while nonautologous included allogenic cadaveric bone from a tissue bank. Revision surgery was defined as a lumbar fusion procedure using osteobiologic grafts during a readmission following a primary admission for lumbar fusion with osteobiologic graft material. Patients with ICD-10 codes for more than 1 osteobiologic type were excluded from the analysis such that all patients had only 1 lumbar fusion code (ie, patients did not receive both autologous and nonautologous biologics, etc). After excluding the necessary individuals for 30-, 90-, and 180-day readmissions, all patient demographics in each group along with several common complications (Table 1) were obtained.

\section{Statistical Analysis}

The statistical analysis was conducted in 2 parts: part 1 was for patients receiving lumbar fusion at a single-vertebral level, and part 2 was for patients receiving multilevel lumbar fusion. Using ICD-10 codes, complications, demographic data, and hospital-reported charge information were collected for each readmission and compared across the 3 osteobiologic groups. Statistical analysis was conducted in RStudio. One-way analysis of variance with Tukey multiple comparisons of means was used to compare complications, demographics, and readmission rates between groups as well as hospital-reported charges, length of stay, and other perioperative variables.

\section{RESULTS}

Using the 2016 and 2017 NRD, we identified 125,143 initial admissions for osteobiologic fusion of the lumbar spine at any level.

\section{Single-Level Lumbar Fusion}

\section{Primary Admission}

Of the 125143 initial fusion procedures, 56364 received fusion with autologous graft, 8603 received fusion with nonautologous graft, and 1967 received fusion with synthetic graft material. Thus, one-toone propensity score matching of all patients receiving fusion with autologous, nonautologous, or synthetic single-level graft material resulted in 3 cohorts of 1967 patients matched for age and sex (Table 2).

Differences were found between the total inpatient hospital-reported charges for patients receiving 
Table 2. Propensity matched single-level fusion patient demographics and cost data.

\begin{tabular}{lccc}
\hline Single-Level Fusion & Autologous $(\mathbf{n}=\mathbf{1 9 6 7})$ & Nonautologous $(\mathbf{n}=\mathbf{1 9 6 7 )}$ & Synthetic $(\mathbf{n}=\mathbf{1 9 6 7})$ \\
\hline Age, mean $\pm \mathrm{SD}, \mathrm{y}$ & $61.17 \pm 0.38$ & $61.40 \pm 2.39$ & $61.16 \pm 13.44$ \\
Female, \% & 55.3 & 57.5 & 54.4 \\
Total hospital-reported charges, mean $\pm \mathrm{SD}, \$$ & $115331.50 \pm 86082.66$ & $149043.70 \pm 118950.90$ & $143736.70 \pm 152715.10$ \\
Length of stay, mean \pm SD, d & $3.82 \pm 4.45$ & $3.99 \pm 5.42$ & $4.27 \pm 6.84$ \\
Rate of acute posthemorrhagic anemia, \% & 11.29 & 10.12 & 10.83 \\
Rate of immediate postsurgical infection, \% & 0.66 & 1.07 & 1.32 \\
\hline
\end{tabular}

fusion with autologous and nonautologous graft material (autologous: $\$ 115331.50 \pm \$ 86082.66$; nonautologous: $\$ 149043.70 \pm \$ 118$ 950.90, $P<$ .0001 ) and those receiving fusion with autologous and synthetic graft material (autologous: $\$ 115331.50 \pm \$ 86082.66$; synthetic: $\$ 143736.70$ $\pm \$ 152715.10, P<.0001)$, with the total inpatient accrued charges of patients receiving fusion with single-level autologous graft material costing significantly less than its counterparts. The length of stay of patients receiving fusion with autologous graft material was also found to be significantly less than those receiving synthetic grafts (autologous: $3.82 \pm$ 4.45; synthetic: $4.27 \pm 6.84, P=.032)$.

\section{Postoperative Complications}

Postoperative complications were compiled for patients readmitted in 30, 90, and 180 days, as shown in Figure 1 and Table 3. Acute postsurgical hemorrhagic anemia and infection rates were not found to be significantly different between the 3 cohorts. For patients readmitted within 30 days, those who initially received fusion with autologous graft material had higher rates of postoperative pain compared with those who received nonautologous grafts (autologous: $0.57 \%$; nonautologous: $0.17 \%$, $P=.035$ ). Furthermore, patients who received fusion with synthetic graft material had significantly higher rates of neurological injury when compared with those receiving autologous grafts at 30 days (autologous: $0.0 \%$; synthetic: $0.41 \%, P=.044$ ). Rates of revision surgery, urinary tract infection (UTI), hardware failure, pneumonia, and infection were not significant between the 3 osteobiologic groups at 30 days. For patients readmitted within 90 days, those who initially received fusion with autologous graft material had higher rates of postoperative pain compared with those who received nonautologous graft material (autologous: $0.65 \%$; nonautologous: $0.14 \%, P=.027$ ). The same trend in postoperative pain was found for patients readmitted within 180 days (autologous: $0.66 \%$; nonautologous: $0.0 \%, P=.010)$. No significant differences were found in revision surgery, neurological injury, and hardware failure at 90- and 180day follow-up.

\section{Readmissions}

At 30 days, single-level lumbar fusion with autologous, nonautologous, and synthetic graft material had readmission rates of $3.69 \%, 5.15 \%$, and $4.75 \%$,

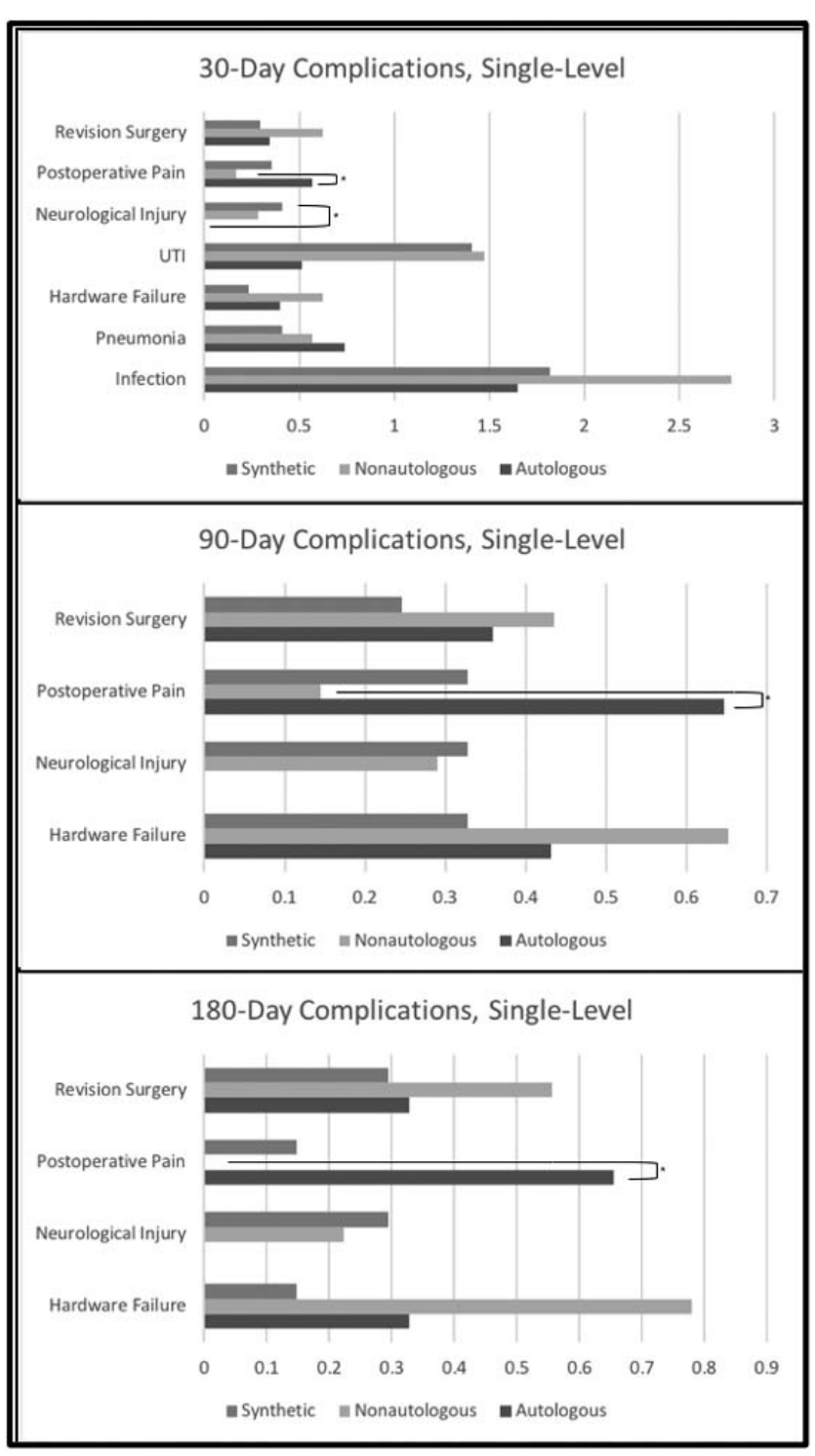

Figure 1. Postoperative complications in single-level lumbar fusion. 
Osteobiologic Subtype Efficacy in Lumbar Fusion

Table 3. Percentage of complication and readmission rates for single-level osteobiologic fusion procedures.

\begin{tabular}{|c|c|c|c|c|c|c|c|}
\hline & Infection & Pneumonia & Hardware Failure & UTI & Neurological Injury & Postoperative Pain & Revision Surgery \\
\hline \multicolumn{8}{|c|}{ 30-day readmissions (excluding December) } \\
\hline Autologous & 1.65 & 0.74 & 0.40 & 0.51 & 0.00 & 0.57 & 0.34 \\
\hline Nonautologous & 2.77 & 0.57 & 0.62 & 1.47 & 0.28 & 0.17 & 0.62 \\
\hline Synthetic & 1.82 & 0.41 & 0.23 & 1.41 & 0.41 & 0.35 & 0.29 \\
\hline \multicolumn{8}{|c|}{ 90-day readmissions (excluding October, November, December) } \\
\hline Autologous & & & 0.43 & & 0.00 & 0.65 & 0.36 \\
\hline Nonautologous & & & 0.65 & & 0.29 & 0.14 & 0.43 \\
\hline Synthetic & & & 0.33 & & 0.33 & 0.33 & 0.25 \\
\hline \multicolumn{8}{|c|}{ 180-day readmissions (excluding July-December) } \\
\hline Autologous & & & 0.33 & & 0.00 & 0.66 & 0.33 \\
\hline Nonautologous & & & 0.78 & & 0.22 & 0.00 & 0.56 \\
\hline Synthetic & & & 0.15 & & 0.29 & 0.15 & 0.29 \\
\hline
\end{tabular}

Abbreviation: UTI, urinary tract infection.

respectively. At 90 days, single-level lumbar fusion with autologous, nonautologous, and synthetic graft material had readmission rates of $6.96 \%, 8.33 \%$, and $8.42 \%$, respectively. At 180 days, single-level lumbar fusion with autologous, nonautologous, and synthetic graft material had readmission rates of $8.95 \%, 10.68 \%$, and $9.59 \%$, respectively. Readmission rates were not significant between any of the 3 osteobiologic groups at 30, 90, and 180 days.

\section{Multilevel Lumbar Fusion}

\section{Primary Admission}

Of the 125143 initial fusion procedures, 48950 received multilevel fusion with autologous graft material, 7602 received multilevel fusion with nonautologous graft material, and 1657 received multilevel fusion with synthetic graft material. Thus, one-to-one propensity score matching of all patients receiving multilevel lumbar fusion with autologous, nonautologous, or synthetic graft material resulted in 3 cohorts of 1657 patients matched for age and sex (Table 4). Differences in total inpatient hospitalacquired charges were found between all 3 groups, with patients receiving fusion with nonautologous graft material having significantly more expensive inpatient hospital charges than those receiving both autologous (nonautologous: \$221 584.40 \pm \$181376.00; autologous: \$182609.10 \pm $\$ 152830.90, P<.0001)$ and synthetic (nonautolo- gous: $\$ 221584.40 \pm \$ 181376.00$; synthetic: $\$ 202166.70 \pm \$ 215914.00, P<.01)$ graft material. In addition, the total inpatient charges associated with fusions involving synthetic graft material were significantly more expensive than those involving autologous graft material (synthetic: \$202 $166.70 \pm$ \$215914.00; autologous: \$182609.10 \pm $\$ 152830.90, P<.01)$. Fusion with autologous graft material was found to have a significantly lower inpatient length of stay compared with fusion with both nonautologous (autologous: $5.47 \pm 5.75$; nonautologous: $6.70 \pm 8.64, P<.0001)$ and synthetic (autologous: $5.47 \pm 5.75$; synthetic: 6.39 $\pm 8.64, P=.0019)$ graft material.

\section{Postoperative Complications}

Postoperative complications were compiled for patients readmitted in 30, 90, and 180 days, as shown in Figure 2 and Table 5. Fusion with autologous graft material had a significantly higher rate of acute posthemorrhagic anemia when compared with fusion with nonautologous (autologous: $24.92 \%$; nonautologous: $10.94 \%, P=.016)$ and synthetic (autologous: $24.92 \%$; synthetic: $21.06 \%, P$ $=.021$ ) graft material, and had a significantly lower postsurgical infection rate when compared with fusion with nonautologous graft material (autologous: $1.45 \%$; nonautologous: $3.26 \%, P=.0020$ ). For patients readmitted within 30 days, those who initially received fusion with autologous graft

Table 4. Propensity matched multilevel fusion patient demographics and cost data.

\begin{tabular}{lccc}
\hline & Autologous $(\mathbf{n}=\mathbf{1 6 5 7})$ & Nonautologous $(\mathbf{n}=\mathbf{1 6 5 7})$ & Synthetic $(\mathbf{n}=\mathbf{1 6 5 7})$ \\
\hline Age, mean $\pm \mathrm{SD}, \mathrm{y}$ & $61.88 \pm 0.33$ & $61.66 \pm 2.30$ & $61.51 \pm 15.82$ \\
Female, \% & 52.1 & 54.0 & 52.3 \\
Total hospital-reported charges, mean $\pm \mathrm{SD}, \$$ & $182609.10 \pm 152830.90$ & $221584.40 \pm 181376.00$ & $202166.70 \pm 215914.00$ \\
Length of stay, mean \pm SD, d & $5.47 \pm 5.75$ & $6.70 \pm 8.64$ & $6.39 \pm 8.64$ \\
Rate of acute posthemorrhagic anemia, \% & 24.92 & 10.94 & 21.06 \\
Rate of immediate postsurgical infection, \% & 1.45 & 3.26 & 2.53 \\
\hline
\end{tabular}




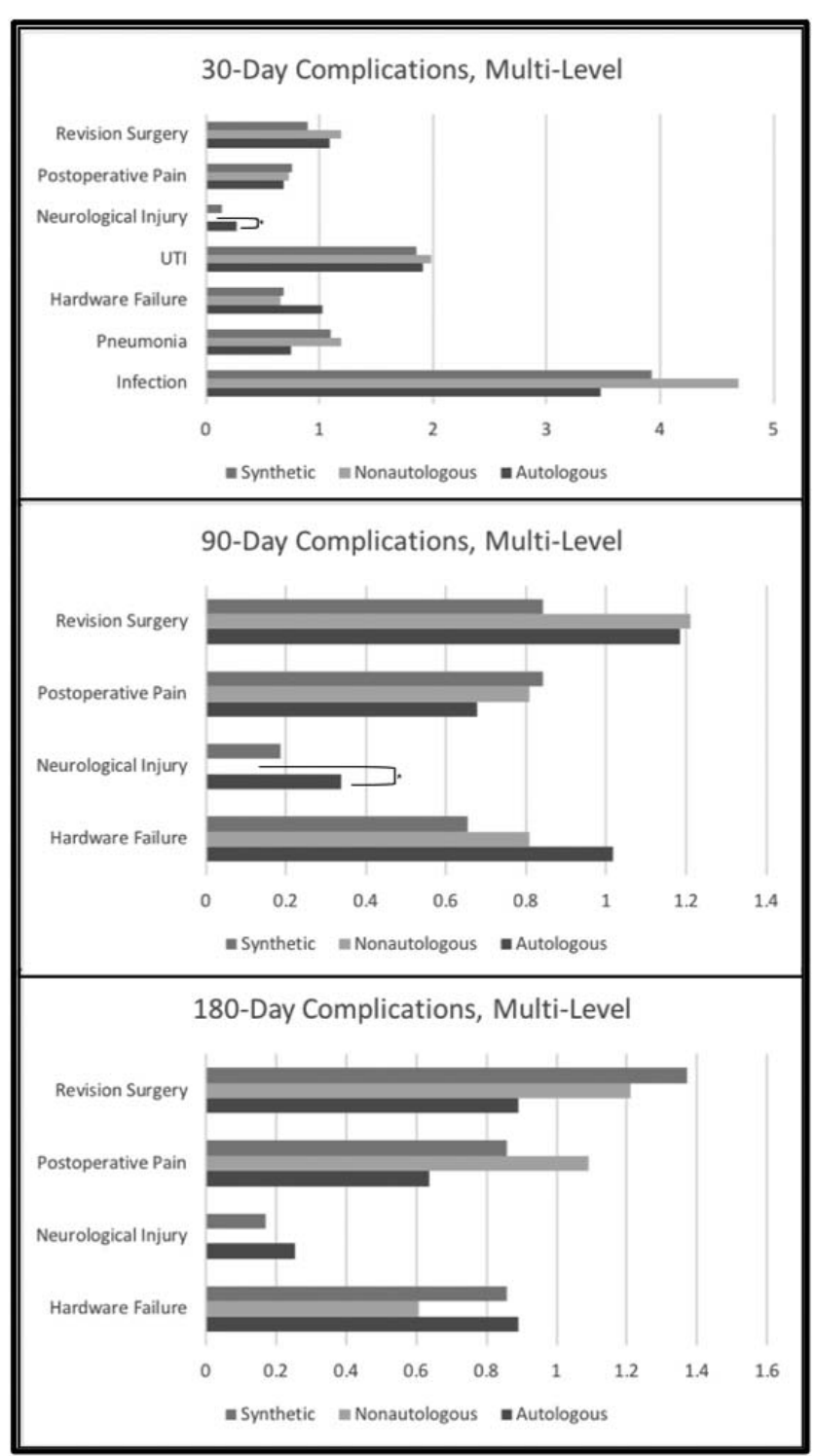

Figure 2. Postoperative complications in multilevel lumbar fusion.

material had higher rates of neurological injury compared with those who received fusion with nonautologous graft material (autologous: 0.27\%; nonautologous: $0.0 \%, P=.049$ ). Rates of postop- erative pain, revision surgery, UTI, hardware failure, pneumonia, and infection were not significant between the 3 osteobiologic groups at 30 days. For patients readmitted within 90 days, those who initially received fusion with autologous graft material also had higher rates of neurological injury compared with those who received fusion with nonautologous graft material (autologous: $0.34 \%$; nonautologous: $0.0 \%, P=.048$ ). However, this trend in neurological injury was not found between osteobiologic groups at 180 days (autologous: $0.25 \%$; nonautologous: $0.0 \%, P=.18$ ). No significant differences were found in revision surgery, postoperative pain, and hardware failure at $90-$ and 180-day follow-up.

\section{Readmissions}

At 30 days, multilevel fusion with autologous, nonautologous, and synthetic lumbar graft material had readmission rates of $5.86 \%, 6.41 \%$, and $6.95 \%$, respectively. At 90 days, multilevel fusion with autologous, nonautologous, and synthetic lumbar graft material had readmission rates of $10.08 \%$, $11.47 \%$, and $11.32 \%$, respectively. At 180 days, multilevel fusion with autologous, nonautologous, and synthetic lumbar graft material had readmission rates of $11.31 \%, 16.85 \%$, and $14.58 \%$, respectively. A significant difference in readmission rates was found between those receiving fusion with autologous and nonautologous graft material at 180-day follow-up, with nonautologous graft patients having a significantly increased readmission rate $(P=$ $.0042)$.

\section{DISCUSSION}

Current study used propensity-matched patients who received single-level and multilevel lumbar fusion across either autologous, nonautologous, or

Table 5. Percentage of complication and readmission rates for multilevel osteobiologic fusion procedures.

\begin{tabular}{|c|c|c|c|c|c|c|c|}
\hline & Infection & Pneumonia & Hardware Failure & UTI & Neurological Injury & Postoperative Pain & Revision Surgery \\
\hline \multicolumn{8}{|c|}{ 30-day readmissions (excluding December) } \\
\hline Autologous & 3.47 & 0.75 & 1.02 & 1.91 & 0.27 & 0.68 & 1.09 \\
\hline Nonautologous & 4.69 & 1.19 & 0.66 & 1.98 & 0.00 & 0.73 & 1.19 \\
\hline Synthetic & 3.92 & 1.10 & 0.69 & 1.86 & 0.14 & 0.76 & 0.89 \\
\hline \multicolumn{8}{|c|}{ 90-day readmissions (excluding October, November, December) } \\
\hline Autologous & & & 1.02 & & 0.34 & 0.68 & 1.19 \\
\hline Nonautologous & & & 0.81 & & 0.00 & 0.81 & 1.21 \\
\hline Synthetic & & & 0.65 & & 0.19 & 0.84 & 0.84 \\
\hline \multicolumn{8}{|c|}{ 180-day readmissions (excluding July-December) } \\
\hline Autologous & & & 0.89 & & 0.25 & 0.64 & 0.89 \\
\hline Nonautologous & & & 0.61 & & 0.00 & 1.09 & 1.21 \\
\hline Synthetic & & & 0.86 & & 0.17 & 0.86 & 1.37 \\
\hline
\end{tabular}

Abbreviation: UTI, urinary tract infection. 
synthetic graft material, and our results add a unique perspective to the prior literature regarding the efficacy of various osteobiologic materials. The major finding of this study was that single-level fusion with autologous graft material resulted in significantly more postoperative pain diagnoses at 30-, 90-, and 180-day follow-up compared with single-level fusion with nonautologous graft material. Multilevel fusion with autologous graft material was found to have higher rates of acute postsurgical hemorrhagic anemia compared with fusion with synthetic and nonautologous alternatives, but also found to have a lower rate of immediate postsurgical infection when compared with that of nonautologous graft material. In addition, lumbar fusion procedures using autologous graft material were associated with significantly more postoperative neurological complications at 30- and 90-day follow-up for multilevel fusion and at 30-day follow-up for single-level fusion compared with fusion with nonautologous graft material. Multilevel fusion with autologous graft material was also found to have a significantly lower nonelective readmission rate compared with fusion with nonautologous graft material at 180-day follow-up. However, significant differences were seen in the total inpatient hospital-reported charges between the 3 osteobiologic subtypes for both single-level and multilevel, with inpatient hospital courses of patients receiving nonautologous graft material being the most expensive and inpatient hospital courses of patients receiving autologous graft material being the least expensive.

A large impetus driving the development of novel synthetic and nonautologous osteobiologic material stems from the desire to avoid autologous tissue harvesting, thus sparing damage to iliac crests or other graft sites. ${ }^{23,24}$ In the current study, fusions with autologous graft had higher rates of acute posthemorrhagic anemia, increased postoperative pain, neurological injury, and readmissions. This is in disagreement with the large study review conducted by Kadam et $\mathrm{al}^{21}$ which independently found no significant differences in the reported clinical outcomes across all classes of bone substitutes. This discrepancy may be attributable to our usage of propensity matching. In addition, Campana et $\mathrm{al}^{25}$ emphasizes the potentially safe nature of approved osteobiologics and highlights differences in efficacy and cost between the osteobiologic groups. Although we lack full 1-year follow-up, the lack of significance between osteobiologic subtypes at 180 days suggests that all 3 osteobiologic might be efficacious. Such a finding is consistent with a 2013 study by Kelly et al, ${ }^{26}$ which found no changes in the rate of revision surgery in spinal deformity patients from 1995-2008 despite the advancement and introduction of novel osteobiologics. Lastly, the same study describes significant improvements in self-reported pain scores after osteobiologic fusion, whereas our study suggests that postoperative pain continues in patients at 30-, 90-, and 180-day follow-up. ${ }^{26}$ Additional multicenter studies that follow osteobiologic fusion patients for several years are required to confirm this observation.

In the current study hospital-reported charges analysis suggests that patients treated with synthetic osteobiologics for single-level fusion pay an average of \$28 405.20 more over the course of their hospitalization compared with single-level fusion with autologous graft material. Patients treated with single-level fusion with nonautologous graft material pay an average of $\$ 33712.20$ more than singlelevel autologous fusion patients over the course of their hospitalization, and $\$ 5307.00$ more than single-level synthetic fusion patients. These trends were also found for multilevel fusion and are reflective of the relative hospital-reported charges and comparisons cited in the literature. The high cost of nonautologous bone grafts, namely allogeneic/allografts, is a widely recognized limitation. ${ }^{6}$ Much of these costs come from maintenance and storage of graft material after a harvest procedure, sterilization of the graft, treatment of graft material for storage, all of which are translated to substantial costs. ${ }^{27}$ For example, 1 study found that in patients who had undergone anterior cervical discectomy/ fusion, the cost of autologous iliac grafts was $\$ 0.00$, whereas the cost of allografts was up to $\$ 2552$ per graft excluding overhead costs. ${ }^{28}$ Haws et $\mathrm{al}^{29}$ found the direct cost of autologous iliac crest bone graft (\$19 315) to be significantly less than bone morphogenic protein (\$21 645) in lumbar fusions. The average length of stay adjusted hospital-reported charges of inpatient stay followed the same trend between the 3 osteobiologic groups. Statistical analysis in this study also confirmed that these differences in prices are significant. However, all patients treated with osteobiologic fusion showed similar postoperative complication rates and timesto-readmission regardless of osteobiologic type, 
which brings up questions about whether certain nonautologous and synthetic biologics are worth their associated higher hospital-reported charges.

\section{Limitations}

This study has several limitations. Firstly, there are inherent limitations associated with a retrospective database study including a dependence on the quality and depth of the records kept for patients included in this study. It is also limited by its retrospective analysis during a narrow range of time (2016 and 2017 only). However, the dates were chosen due to the implementation of mandatory ICD-10 coding in late 2015, which allowed for more detailed codes from which to draw data. Thus, despite the limited time window and decreased potential sample size, this study was able to achieve greater granularity than any previous studies that used ICD-9 codes. Another study limitation is that the NRD encapsulates roughly $60 \%$ of the US population, so the conclusions drawn in this study may not be widely representative of the entire US population. Using a national, deidentified database, we also lack patient-reported outcomes of pain and disability following each surgical procedure as these outcomes lack specific ICD-10 codes. This study also did not analyze the differences in elective readmissions between groups, which may be related to poor patient-related outcomes following use of 1 or multiple types of osteobiologic. Additionally, the study did not assess fusion rates, pseudarthrosis rates, or costs associated with management of symptomatic pseudarthrosis. Finally, the specificity of our osteobiologic subtyping is limited by the ICD-10 codes available for osteobiologics. For example, although autologous osteobiologics are generally homogeneous, there are multiple different allografts and synthetic osteobiologics on the market are combined into a single group due to ICD coding. Further studies are required to analyze and compare the efficacy of individual synthetic osteobiologics, which may be highly heterogeneous.

\section{CONCLUSION}

This study used a large national readmission database to identify and propensity match patients who received single-level and multilevel lumbar fusion across either autologous, nonautologous, or synthetic osteobiologics. Multilevel fusion with autograft had higher rates of acute postsurgical hemorrhagic anemia compared with synthetic and nonautologous alternative, and a lower rate of immediate postsurgical infection when compared with that of fusion with nonautologous graft. We also found that fusion with autograft resulted in significantly more postoperative pain diagnoses for single-level fusion at all follow-up timepoints and significantly more postoperative neurological complications at 30- and 90-day follow-up for multilevel fusion. At 180-day follow-up, multilevel fusion with autologous graft was found to have a significantly lower nonelective readmission rate compared with fusion with the nonautologous graft. Fusion with nonautologous graft was found to be more expensive than procedures with autologous and synthetic material. Future randomized controlled trials with long-term follow-up are needed to assess the effects of the different osteobiologics.

\section{REFERENCES}

1. Hsu WK, Goldstein CL, Shamji MF, et al. Novel osteobiologics and biomaterials in the treatment of spinal disorders. Neurosurgery. 2017;80(3S):S100-S107.

2. Yoo JS, Ahn J, Patel DS, Hrynewycz NM, Brundage TS, Singh K. An evaluation of biomaterials and osteobiologics for arthrodesis achievement in spine surgery. Ann Transl Med. 2019;7(Suppl 5):S168.

3. Lauweryns P, Raskin Y. Prospective analysis of a new bone graft in lumbar interbody fusion: results of a 2- year prospective clinical and radiological study. Int J Spine Surg. 2015;9. doi:10.14444/2002

4. Glassman SD, Howard JM, Sweet A, Carreon LY. Complications and concerns with osteobiologics for spine fusion in clinical practice. Spine. 2010;35(17):1621-1628.

5. Buser Z, Brodke DS, Youssef JA, et al. Synthetic bone graft versus autograft or allograft for spinal fusion: a systematic review. J Neurosurg Spine. 2016;25(4):509-516.

6. Fernandez de Grado G, Keller L, Idoux-Gillet Y, et al. Bone substitutes: a review of their characteristics, clinical use, and perspectives for large bone defects management. J Tissue Eng. 2018;9:2041731418776819.

7. Dimitriou R, Mataliotakis GI, Angoules AG, Kanakaris NK, Giannoudis PV. Complications following autologous bone graft harvesting from the iliac crest and using the RIA: a systematic review. Injury. 2011;42(Suppl 2):S3-S15.

8. Chen W-J, Tsai T-T, Chen L-H, et al. The fusion rate of calcium sulfate with local autograft bone compared with autologous iliac bone graft for instrumented short-segment spinal fusion. Spine. 2005;30(20):2293-2297.

9. An HS, Lynch K, Toth J. Prospective comparison of autograft vs. allograft for adult posterolateral lumbar spine fusion: differences among freeze-dried, frozen, and mixed grafts. J Spinal Disord. 1995;8(2):131-135.

10. Fishman JA, Greenwald MA, Grossi PA. Transmission of infection with human allografts: essential considerations in donor screening. Clin Infect Dis. 2012;55(5):720-727.

11. Khan SN, Cammisa FP Jr, Sandhu HS, Diwan AD, 
Girardi FP, Lane JM. The biology of bone grafting. J Am Acad Orthop Surg. 2005;13(1):77-86.

12. Hsieh PC, Buser Z, Skelly AC, et al. Allogenic stem cells in spinal fusion: a systematic review. Global Spine J. 2019;9(1 Suppl):22S-38S.

13. Khashan M, Inoue S, Berven SH. Cell based therapies as compared to autologous bone grafts for spinal arthrodesis. Spine. 2013;38(21):1885-1891.

14. Ehrler DM, Vaccaro AR. The use of allograft bone in lumbar spine surgery. Clin Orthop Relat Res. 2000;(371):38-45.

15. Glassman SD, Carreon L, Djurasovic M, et al. Posterolateral lumbar spine fusion with INFUSE bone graft. Spine J. 2007;7(1):44-49.

16. Lee K-B, Taghavi CE, Hsu MS, et al. The efficacy of rhBMP-2 versus autograft for posterolateral lumbar spine fusion in elderly patients. Eur Spine J. 2010;19(6):924-930.

17. Glassman SD, Carreon LY, Campbell MJ, et al. The perioperative cost of Infuse bone graft in posterolateral lumbar spine fusion. Spine J. 2008;8(3):443-448.

18. Tannoury CA, An HS. Complications with the use of bone morphogenetic protein 2 (BMP-2) in spine surgery. Spine J. 2014;14(3):552-559.

19. Epstein NE. Complications due to the use of BMP/ INFUSE in spine surgery: the evidence continues to mount. Surg Neurol Int. 2013;4(Suppl 5):S343-S352.

20. Fu R, Selph S, McDonagh M, et al. Effectiveness and harms of recombinant human bone morphogenetic protein-2 in spine fusion: a systematic review and meta-analysis. Ann Intern Med. 2013;158(12):890-902.

21. Kadam A, Millhouse PW, Kepler CK, et al. Bone substitutes and expanders in spine surgery: a review of their fusion efficacies. Int J Spine Surg. 2016;10:33.

22. World Health Organization. International Classification of Diseases, Tenth Revision. Geneva: WHO; 2010.

23. Thaler M, Lechner R, Gstöttner M, Kobel C, Bach C. The use of beta-tricalcium phosphate and bone marrow aspirate as a bone graft substitute in posterior lumbar interbody fusion. Eur Spine J. 2013;22(5):1173-1182. doi:10.1007/s00586-0122541-3

24. Yamada T, Yoshii T, Sotome S, Yuasa M, Kato T, Arai Y. Hybrid grafting using bone marrow aspirate combined with porous $\beta$-tricalcium phosphate and trephine bone for lumbar posterolateral spinal fusion: a prospective, comparative study versus local bone grafting. Spine. 2012;37(3):E174-E179.

25. Campana V, Milano G, Pagano E, et al. Bone substitutes in orthopaedic surgery: from basic science to clinical practice. $J$ Mater Sci Mater Med. 2014;25(10):2445-2461.

26. Kelly MP, Lenke LG, Bridwell KH, Agarwal R, Godzik J, Koester L. Fate of the adult revision spinal deformity patient. Spine. 2013;38(19):E1196-E1200. doi:10.1097/brs. $0 \mathrm{~b} 013 \mathrm{e} 31829 \mathrm{e} 764 \mathrm{~b}$

27. Russell JL, Block JE. Surgical harvesting of bone graft from the ilium: point of view. Med Hypotheses. 2000;55(6):474479.
28. Epstein NE. Efficacy and outcomes of dynamic-plated single-level anterior diskectomy/fusion with additional analysis of comparative costs. Surg Neurol Int. 2011;2:9.

29. Haws BE, Khechen B, Narain AS, et al. Iliac crest bone graft for minimally invasive transforaminal lumbar interbody fusion: a prospective analysis of inpatient pain, narcotics consumption, and costs. Spine. 2018;43(18):1307-1312.

Disclosures and COI: There are no conflicts of interest in this study. There are also no disclosures of funding for this study. No patient identifiers were collected. Disclosures outside of submitted work: JCW: Royalties-Biomet, SeaSpine, Amedica, Synthes; Investments/OptionsBone Biologics, Pearldiver, Electrocore, Surgitech; Board of Directors-AO Foundation, Society for Brain Mapping and Therapeutics, American Orthopaedic Association; Editorial Boards-Spine, The Spine Journal, Clinical Spine Surgery, Global Spine Journal; Fellowship Funding (paid to institution): AO Foundation; ZB: Consultancy-Cerapedics (past), The Scripps Research Institute (past), Xenco Medical (past), AO Spine (past); Research Support-SeaSpine (past, paid to the institution), Next Science (paid directly to institution), Motion Metrics (paid directly to institution); North American Spine Society-committee member; Lumbar Spine Society-Co-chair Education committee, AO Spine Knowledge Forum Degenerative: Associate member; AOSNA Research committee-committee member.

Corresponding Authors: Zorica Buser, $\mathrm{PhD}$ and Shane Shahrestani, MS, Department of Orthopaedic Surgery, Keck School of Medicine, University of Southern California, Los Angeles, CA. Phone: (323) 442-0206; Emails: shanesha@usc.edu; zbuser@usc.edu.

Published 19 August 2021

This manuscript is generously published free of charge by ISASS, the International Society for the Advancement of Spine Surgery. Copyright (C) 2021 ISASS. To see more or order reprints or permissions, see http://ijssurgery.com. 